04

\title{
Электрические токи, индуцированные в плазме ионно-звуковыми солитонами: учет захваченных электронов
}

\author{
(C) Ф.М. Трухачев ${ }^{1,2}$, А.В. Томов ${ }^{3}$, М.М. Могилевский ${ }^{4}$, \\ Д.В. Чугунин ${ }^{4}$
}

${ }^{1}$ Белорусско-Российский университет, Могилев, Беларусь

${ }^{2}$ Объединенный институт высоких температур РАН, Москва, Россия

${ }^{3}$ Могилевский государственный университет, Могилев, Беларусь

${ }^{4}$ Институт космических исследований РАН, Москва, Россия

E-mail: ftru@mail.ru

Поступило в Редакцию 3 ноября 2017 г.

Исследованы токи, индуцируемые ионно-звуковыми солитонами в двухкомпонентной плазме, в приближении магнитной гидродинамики (МГД-модели) с учетом захваченных электронов. Показано, что солитоны возбуждают однополярные импульсы ионного и электронного токов, описаны механизмы их возбуждения. Рассчитаны пространственно-временны́е характеристики токовых импульсов, определены требования к пространственно-временно́му разрешению экспериментального оборудования, необходимого для регистрации плазменных токов, индуцированных солитонами. Показано, что солитоны являются эффективным механизмом генерации плазменных токов.

DOI: 10.21883/PJTF.2018.11.46201.17110

Основные свойства ионно-звуковых солитонов были детально изучены к началу 80-х годов прошлого века [1]. Тем не менее ряд вопросов остается нерешенным до сих пор. Одним из них является вопрос об электрических токах, возбуждаемых электрическими полями солитонов в плазме. Задачу можно свести к анализу движения заряженных частиц, формирующих солитон. Вопрос о движении частиц холодных популяций в электрических полях ионно-звуковых солитонов был решен в [2]. Исследования были мотивированы необходимостью определения пространственно-временно́го разрешения токоизмерительной аппаратуры для регистрации солитонов в космосе, актуальность указанной задачи отмечена в [3]. В приближении магнитной гидродинамики 
(МГД-модели) в [2] было показано, что солитоны могут осуществлять однонаправленный перенос заряженных частиц на значительное расстояние; установлено также, что среднее значение (постоянная составляющая) ионного тока, обусловленного движением ансамбля солитонов, по величине сопоставимо с током, создаваемым пучками ионов. Выводы [2] были экспериментально подтверждены в пылевой плазме [4], которая представляет интерес как с технологической, так и с научной точки зрения [5]. В настоящей работе модель [2] обобщена с учетом захваченных электронов, что позволяет рассчитать движение всех плазменных популяций и, следовательно, более точно описать поведение реальной плазмы.

Для определения направления потоков, формирующих возмущение плотности и заряда в солитоне, рассмотрим модель водородной плазмы при $T_{e}>>T_{i}$ (где $T_{e}, T_{i}$ - температуры электронов и ионов соответственно), в которой можно пренебречь затуханием Ландау для ионнозвуковых волн. Такая плазма наблюдается как в лаборатории [1,5], так и в отдельных областях космоса [6]. Как показано в [7], корректный анализ солитонов требует учета захваченных электронов. Ионно-звуковой солитон сжатия является для электронов потенциальной ямой $[7,8]$, в которой часть из них совершает колебательное движение. Такие электроны двигаются вместе с солитоном и называются захваченными в отличие от пролетных, которые находятся в яме конечное время. Воспользуемся МГД-моделью [7]

$$
\begin{gathered}
\frac{\partial v_{i}}{\partial t}+v_{i} \frac{\partial v_{i}}{\partial x}=-\frac{e}{m_{i}} \frac{\partial \varphi}{\partial x}, \\
\frac{\partial n_{i}}{\partial t}+\frac{\partial n_{i} v_{i}}{\partial x}=0, \\
n_{e}=n_{0} \exp \left(\frac{e \varphi}{T_{e}}\right)\left[1-\operatorname{erf}\left(\sqrt{\frac{e \varphi}{T_{e}}}\right)\right]+2 n_{0} \sqrt{\frac{e \varphi}{\pi T_{e}}}, \\
\frac{\partial^{2} \varphi}{\partial x^{2}}=-\frac{e}{\varepsilon_{0}}\left(n_{i}-n_{e}\right) .
\end{gathered}
$$

Здесь $v_{i}$ - скорость ионов, $n_{i}, n_{e}$ - концентрации ионов и электронов, $n_{0}$ - невозмущенная концентрация заряженных частиц, $m_{i}-$ масса ионов, $e-$ элементарный заряд, $\varphi-$ электрический потенциал, 
соответствующий электростатическому полю $E=-\partial \varphi / \partial x$. В рассматриваемом простейшем случае $T_{i} \approx 0, p_{i} \approx 0$, где $p_{i}-$ ионное давление. При $T_{i}>0$, согласно [1], в уравнение (1) нужно добавить член, учитывающий $p_{i}$. Ограничимся поиском стационарных решений системы (1)-(4), выполнив замену переменных $X=(x-V t) / \lambda_{\mathrm{D}}$, где $V$ - установившаяся скорость солитона, $\lambda_{\mathrm{D}}=\sqrt{\varepsilon_{0} T_{e} / e^{2} n_{0}}-$ радиус Дебая. Введем нормировку для концентраций $N_{i}=n_{i} / n_{0}, N_{e}=n_{e} / n_{0}$ и потенциала $\Phi=e \varphi / T_{e}$, амплитуду солитонов обозначим $\Phi_{0}$. Выражение для $N_{i}$ можно записать в виде [2]:

$$
N_{i}(\Phi)=M / \sqrt{M^{2}-2 \Phi}
$$

где $M=V / C$ - число Маха, $C=\sqrt{T_{e} / m_{i}}-$ ионно-звуковая скорость. Уравнения (3), (4) с учетом нормировки примут вид

$$
\begin{gathered}
N_{e}(\Phi)=\exp (\Phi)[1-\operatorname{erf}(\sqrt{\Phi})]+2 \sqrt{\Phi / \pi} \\
\frac{d^{2} \Phi}{d X^{2}}=N_{e}(\Phi)-N_{i}(\Phi)
\end{gathered}
$$

или

$$
\begin{aligned}
\frac{d^{2} \Phi}{d X^{2}} & =\exp (\Phi)[1-\operatorname{erf}(\sqrt{\Phi})] \\
& +2 \sqrt{\Phi / \pi}-M / \sqrt{M^{2}-2 \Phi}
\end{aligned}
$$

Однократное интегрирование (7) дает выражение для псевдопотенциала Сагдеева

$$
\begin{aligned}
U(\Phi) & =-\frac{1}{2}\left(\frac{d \Phi}{d X}\right)^{2}=-M \sqrt{M^{2}-2 \Phi}-\exp (\Phi)[1-\operatorname{erf}(\sqrt{\Phi})] \\
& -2 \sqrt{\Phi / \pi}-4 \sqrt{\Phi^{3} / 9 \pi}+A
\end{aligned}
$$

где $A$ - константа интегрирования, выбор которой определяет вид решений уравнения (7). Особым классом решений при $A=A_{0}=M^{2}+1$ $(d \Phi / d X=0$ при $\Phi=0)$ являются солитоны. На рис. 1 представлена зависимость $d \Phi / d X$ от $\Phi$ для разных значений $A$ и $M$, из которой можно легко найти амплитуду солитонов и соответствующие граничные условия для численного интегрирования.

Письма в ЖТФ, 2018, том 44, вып. 11 


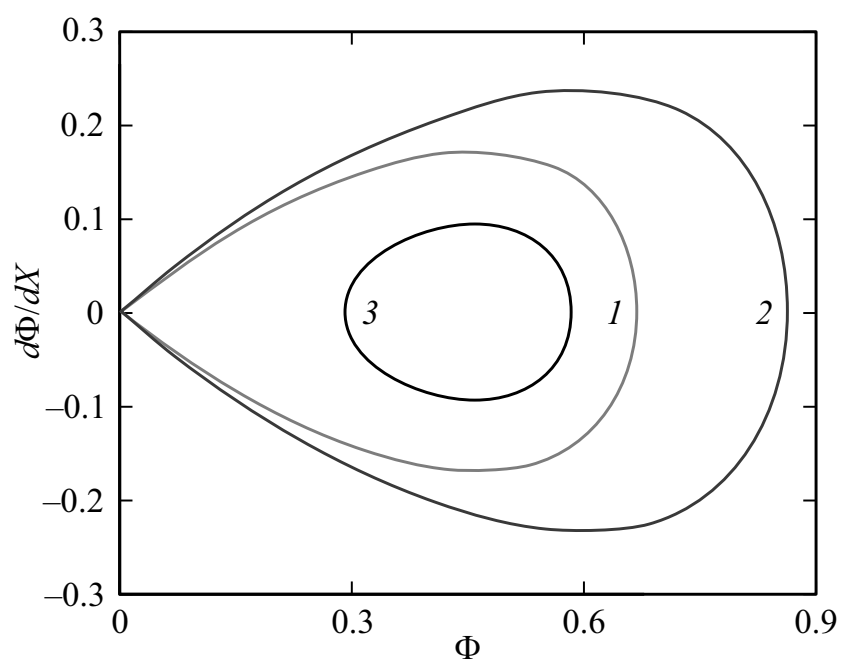

Рис. 1. Фазовая плоскость уравнения (7). $1-M=1.5, A=A_{0} ; 2-M=1.6$, $A=A_{0} ; 3-M=1.5, A=A_{0}+0.01$.

На рис. 1 кривые 1 и 2 отвечают солитонам, кривая 3 - решению в виде периодических нелинейных волн. Профили соответствующего кривой 1 солитона как результат численного решения (8) представлены на рис. 2. Как видно, солитону соответствует повышение концентраций $N_{i}, N_{e}$ (рис. $\left.2, b\right)$, при этом $N_{i} \neq N_{e}$ внутри солитона, но в целом концентрации компенсируют друг друга, так что $\int_{-\infty}^{\infty}\left(N_{i}-N_{e}\right) d X=0$. Для определения скорости ионов воспользуемся формулой $v_{i}=V\left(1-N_{i}^{-1}\right)$ из [2], которую с учетом нормировки на $C$ можно записать в виде $U_{i}=M\left(1-N_{i}^{-1}\right)$.

Профиль ионной скорости $U_{i}(X)$ в поле солитона представлен на рис. $2, a$, из которого видно, что $U_{i}>0$. Это хорошо согласуется с результатами [9], где получены решения МГД-уравнений для скорости ионов. Следовательно, профиль $N_{i}>1$ формируется именно однонаправленным перемещением ионов в локальной области шириной $\sim 10 \lambda_{\mathrm{D}}$. Другими словами, ионы, формирующие профиль солитона, перемещаются только в направлении его распространения (причем 

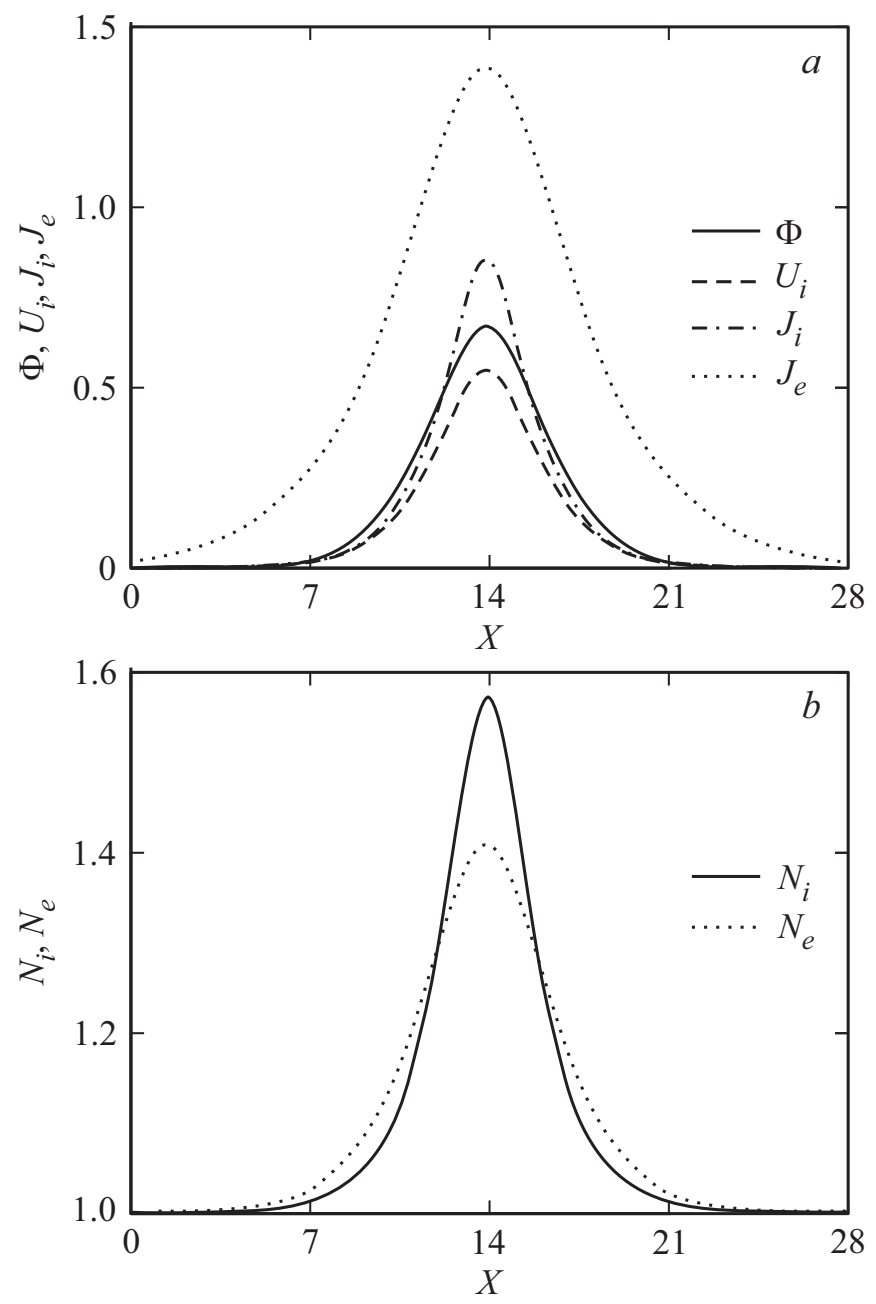

Рис. 2. Ионно-звуковой солитон при $M=1.5 . a-$ профили $\Phi(X), U_{i}(X), J_{i}(X)$, $J_{e}(X) ; b-$ профили $N_{i}(X), N_{e}(X)$.

каждый ион смещается на конечное расстояние [2]). Плотность ионного тока легко получить, умножив $v_{i}$ на $e n_{i}$, в виде $j_{i}=e n_{i} V\left(1-N_{i}^{-1}\right)$ или

Письма в ЖТФ, 2018, том 44, вып. 11 
с нормировкой на $e n_{0} C$

$$
J_{i}=M\left(N_{i}-1\right) .
$$

Профиль $J_{i}(X)$ представлен на рис. 2,a. Как видно, солитон формирует однополярный импульс ионного тока, соразмерный по ширине с импульсом скорости. Результаты анализа ионных токов хорошо согласуются с результатами [2], количественные различия связаны лишь с разным описанием электронов и различием методов решения (4). Электронные токи в [2] не анализировались, остановимся на этом подробнее. В моделях [7,8] пролетные частицы приходят из бесконечности, причем эти потоки из $-\infty$ и $+\infty$ равны, их суммарный ток равен нулю. Следовательно, ненулевой электронный ток определяется только захваченными частицами. Поскольку захваченные электроны движутся вместе с солитоном, плотность электронного тока можно выразить, умножив концентрацию захваченных электронов (второе слагаемое в правой части (3)) на скорость движения солитона и заряд электрона: $j_{e}=2 n_{0} e V \sqrt{e \varphi / \pi T_{e}}$ или с учетом нормировки на $e n_{0} C$

$$
J_{e}=2 M \sqrt{\Phi / \pi}
$$

Профиль $J_{e}(X)$ отображен на рис. $2, a$, из которого очевидны пространственная локализованность и однополярный характер $J_{e}(X)$. В $[10]$ можно найти выражение для плотности электронного тока, полученное из уравнений Власова-Пуассона. Полученное нами выражение (10) содержит меньше параметров, что упрощает его практическое использование.

Профили плотности электронного и ионного токов на первый взгляд различаются лишь количественно, однако механизмы возникновения и распространения токов различаются принципиально. Действительно, ионные токи в рассмотренном случае обусловлены инертностью ионов в электрическом поле солитона [2]; причиной же электронных токов, очевидно, является захват электронов потенциальной ямой солитона, движущегося в пространстве. Другими словами, солитон подобен контейнеру для переноса электронов. Каждый ион переносится на конечное расстояние в направлении движения солитона [2]; электроны же захватываются в области формирования солитона и сопровождают его до распада $[7,8]$.

С экспериментальной точки зрения интерес представляют такие параметры, как ширина $L$ и длительность $\chi$ токовых импульсов, а также

Письма в ЖТФ, 2018, том 44, вып. 11 
средние значения (постоянные составляющие) плотности электронного и ионного токов, генерируемых каскадом солитонов. Величины $L$ и $\chi$ с учетом нормировки соответственно на $\lambda_{\mathrm{D}}$ и $\omega_{i}^{-1}\left(\omega_{i}=\sqrt{e^{2} n_{0} / m_{i} \varepsilon_{0}}-\right.$ ионная плазменная частота) оказываются связанными простым соотношением $L=M \chi$. Из анализа численных решений (8) с учетом (9), (10) следует, что $L$ и $\chi$ монотонно убывают с ростом амплитуды солитонов. Для солитонов большой амплитуды $\Phi_{0}=3$ имеем $L_{i}=0.6, L_{e}=5.4$, $\chi_{i}=0.2, \chi_{e}=2.2$, для надежной экспериментальной регистрации формы таких токовых импульсов разрешение приборов должно быть как минимум на порядок меньше указанных величин.

Солитоны в плазме часто наблюдаются в виде каскадов [3]. В этом случае, как показано в [2], постоянная составляющая плотности ионных токов имеет существенную величину и при недостатке разрешения может трактоваться как ток несуществующих ионных пучков. Рассмотрим подобную ситуацию в рамках построенной модели. На рис. 3, $a$ представлено численное решение (8), полученное при $M=1.5, A=A_{0}+6.7 \cdot 10^{-12}$, описывающее токи, индуцированные каскадом из пяти солитонов. Как видно, каскад солитонов индуцирует пульсирующие электронные и ионные токи с существенными постоянными составляющими $\bar{J}_{e}, \bar{J}_{i}$, которые важно отделить от токов пучков. Регистрация стационарных токов пучков не вызывает трудностей, в то время как мелкомасштабные токи солитонов практически не изучены [3]. Проанализируем постоянную составляющую солитонных токов, которая будет зависеть от $\Phi_{0}$ и частоты следования солитонов $v$. Зависимость $\bar{J}_{i}, \bar{J}_{e}$ от $v$ будет пропорциональной, а их зависимость от $\Phi_{0}$ при постоянной $v$ представлена на рис. $3, b$.

Из рис. $3, b$ видно, что $\bar{J}_{i}$ и $\bar{J}_{e}$ являются величинами одного порядка, при этом $\bar{J}_{e}>\bar{J}_{i}$ для любых $\Phi_{0}$. Отметим, что, согласно [11], число захваченных электронов может быть меньше, чем следует из [7,8], а следовательно, и соотношение между $\bar{J}_{i}$ и $\bar{J}_{e}$ может варьироваться.

Полученные здесь результаты полностью подтверждают выводы [2], обобщают их в части токов горячих популяций заряженных частиц и, на наш взгляд, свидетельствуют о существовании нелинейного механизма возбуждения токов в плазме. Суть механизма, который мы с осторожностью назовем солитонным токовым механизмом, заключается в том, что солитоны и, в особенности, каскады солитонов вызывают крупномасштабные перемещения зарядов и возбуждают значительные

Письма в ЖТФ, 2018, том 44, вып. 11 

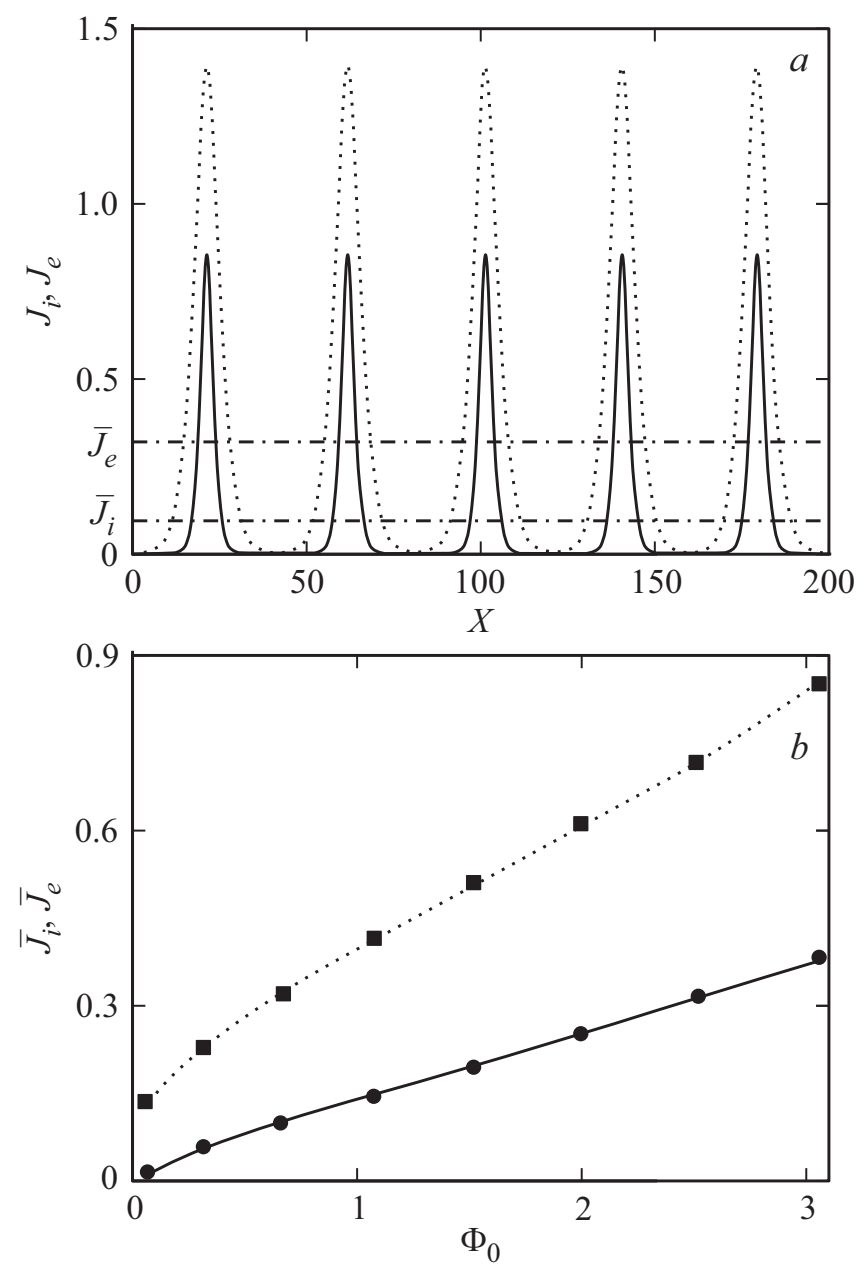

Рис. 3. Ионные (сплошные линии) и электронные (пунктирные линии) токи, индуцированные каскадом из пяти солитонов, при $M=1.5, A=A_{0}+6.7 \cdot 10^{-12}$ и межсолитонном расстоянии $40 \lambda_{\mathrm{D}} . a-$ - зависимость плотности токов от $X$, $b$ - зависимость постоянных составляющих плотности токов от амплитуды солитонов.

Письма в ЖТФ, 2018, том 44, вып. 11 
по величине электронные и ионные токи пульсирующего характера с постоянной составляющей. Полный ток в плазме при этом может быть суперпозицией токов пучков и солитонных токов.

Прикладная значимость работы заключается в определении требований к разрешению экспериментального оборудования для регистрации мелкомасштабной структуры токов в плазме. В более широком смысле полученные результаты могут быть использованы для интерпретации экспериментальных данных по исследованию токов в плазме, в том числе при анализе аномального сопротивления плазмы в условиях турбулентности [12]. Обзор недавних публикаций $[13,14]$ свидетельствует о том, что проблема переноса частиц солитонами не ограничивается плазменными приложениями.

Авторы с благодарностью вспоминают Ю.И. Гальперина, который в свое время внес значительный вклад в постановку задачи по анализу солитонных токов в плазме.

\section{Список литературы}

[1] Tran M.Q. // Phys. Scripta. 1979. V. 20. P. 317-327.

[2] Трухачев Ф.М., Томов А.В. // Космические исследования. 2016. Т. 54 . № 5. C. 377-383.

[3] Pickett J.S., Kahler S.W., Chen L.-J., Huff R.L., Santolik O., Khotyaintsev Y., Décréau P.M.E., Winningham D., Frahm R., Goldstein M.L., Lakhina G.S., Tsurutani B.T., Lavraud B., Gurnett D.A., André M., Fazakerley A., Balogh A., Reme H. // Nonlinear Process. Geophys. 2004. V. 11. P. 183-196.

[4] Трухачев Ф.М., Петров О.Ф., Васильев М.М., Герасименко Н.В. // VI Конгресс физиков Беларуси. Сб. науч. тр. Минск, 2017. С. 281-282.

[5] Фортов В.Е., Храпак А.Г., Храпак С.А., Молотков В.И., Петров О.Ф. // УФН. 2004. Т. 174. № 5. С. 495-544.

[6] Подгорный И.М., Сагдеев Р.3. // УФН. 1969. Т. 98. № 4. С. 410-417.

[7] Гуревич А.В. // ЖЭТФ. 1967. Т. 53. № 3. С. 953-964.

[8] Лифиии, Е.М., Питаевский Л.П. Физическая кинетика. М.: Наука, 1979. $182 \mathrm{c}$.

[9] Johnston C.R., Epstein M. // Phys. Plasmas. 2000. V. 7. P. 906-910.

[10] Алешин И.М., Перегудов Д.В. // Вестн. МГУ. Сер. 3. Физика, астрономия. 2000. № 1. С. $8-11$.

Письма в ЖТФ, 2018, том 44, вып. 11 
[11] Bernstein I.B., Green J.M., Kruskal M.D. // Phys. Rev. 1957. V. 108. P. 546-550.

[12] Сквориова Н.Н., Харчев Н.К., Сарксян К.А. // Письма в ЖЭТФ. 1999. Т. 70. B. 3. C. 203-207.

[13] Poletti D., Ostrovskaya E.A., Alexander T.J., Li B., Kivshar Yu.S. // Physica D. 2009. V. 238. P. $1338-1344$.

[14] Younis M., Ali S. // Appl. Mathem. Comput. 2014. V. 246. P. 460-463. 